\title{
Towards Consensus in Operational Definitions in Functional Capacity Evaluation: a Delphi Survey
}

\author{
Remko Soer - Cees P. van der Schans • \\ Johan W. Groothoff · Jan H. B. Geertzen • \\ Michiel F. Reneman
}

Published online: 15 November 2008

(c) The Author(s) 2008. This article is published with open access at Springerlink.com

\begin{abstract}
Introduction The problem of inconsistent terminology in functional capacity evaluation (FCE) has been widely addressed in the international literature. Many different terms seem to be used interchangeably while other terms appear to be interpreted differently. This may seriously hinder FCE research and clinical use. To gain consensus in operational definitions in FCE and conceptual framework to classify terminology used in FCE. Methods A Delphi Survey with FCE experts was conducted which consisted of three rounds of questioning, using semi and full structured questions. The expert group was formed from international experts in FCE. Experts were selected if they met any of the following criteria: at least one international publication as first author and one as co-author in the field of FCE; or an individual who had developed an FCE that was subject of investigation in at least one publication in international literature. Consensus of definitions was considered when $75 \%$ or more of all experts agreed with a definition. Results In total, 22 international experts from 6 different
\end{abstract}

R. Soer $(\bowtie) \cdot$ J. H. B. Geertzen · M. F. Reneman Center for Rehabilitation, University Medical Center Groningen, University of Groningen, P.O. Box 30.002, 9750 RA Haren, The Netherlands

e-mail: r.soer@cvr.umcg.nl

R. Soer - J. W. Groothoff - J. H. B. Geertzen · M. F. Reneman Graduate School for Health Research, University Medical Center Groningen, University of Groningen, Groningen,

The Netherlands

C. P. van der Schans

Hanze Hogeschool Groningen, University for Professional Education, Groningen, The Netherlands

J. W. Groothoff

Department of Health Sciences, University Medical Center Groningen, Groningen, The Netherlands countries in Australia, Europe and North America, working in different health related sectors, participated in this study. Conclusion Consensus concerning conceptual framework of FCE was met in 9 out of 20 statements. Consensus on definitions was met in 10 out of 19 definitions. Experts agreed to use the ICF as a conceptual framework in which terminology of FCE should be classified and agreed to use pre-defined terms of the ICF. No consensus was reached about the definition of FCE, for which two potential eligible definitions remained. Consensus was reached in many terms used in FCE. For future research, it was recommended that researchers use these terms, use the ICF as a conceptual framework and clearly state which definition for FCE is used because no definition of FCE was consented.

Keywords Work ability - International classification of functioning - Disability and health · ICF · Expert based

\section{Introduction}

Functional capacity evaluations (FCEs) are standardized batteries of tests which all together form an evaluation of capacity of work-related activities. FCEs are used in occupational, insurance, and rehabilitation medicine in order to evaluate work ability. Earlier studies show that there is evidence of reliability and some aspects of validity, depending on the FCE protocol [1]. Worldwide there are multiple FCEs using different protocols from different providers which all claim to measure the same construct, namely functional capacity. However, concurrent validity of these FCE protocols are moderate to poor [2-4]. In addition, when the same protocol is administered in a different environment, different results appear [5]. Differences between various approaches to FCEs may include 
variations in the number of measurements obtained, degree of standardization or the clarity of the concepts and underlying theories [6]. A possible explanation of variation between results, besides the points addressed above, is the lack of consensus used in terms of operational definitions. Different authors have previously addressed this issue $[3,7$, 8]. One study has addressed the problem of confused definitions of terms and confusion in conceptual framework. This study resulted in recommendations on how to use operational definitions in the field of work-related assessments [7]. Additionally, a different study addressed the presumed difference between a kinesiophysical (evaluator terminates a test when maximum is reached) and a psychophysical approach to FCE (patient terminates the test when acceptable maximum is reached). This study, however, found no differences between the test termination criteria and concluded that this presumed difference may be due to a lack of clarity in operational definitions [3]. Others found inconsistencies in terminology in physical functioning, functional ability, physical ability, physical activity, activity, capacity, performance, functional status, functional limitations, etc. and concluded that consensus was needed [7, 8]. Authors have proposed to use the World Health Organizations' International Classification of Functioning, Disability and Health (ICF) to classify workrelated definitions in a world wide consented framework [9-11]. All proposed to use the ICF because it considers functioning as a biopsychosocial understanding of health in which physical and behavioral functions are in dynamic interaction with each other.

The ICF is a classification system which was constructed by the World Health Organization (WHO) and aims to provide a universal classification system of disability and functioning for the use in health and healthrelated sectors. The aims of the ICF are: to provide a scientific basis for understanding and studying health and health-related states, outcomes and determinants; to establish a common language for describing health and health-related states in order to improve communication between different users; and to permit comparison of data across countries, health care disciplines, services and time and to provide a systematic coding scheme for health information systems. The ICF provides a model which describes determinants of functioning which depend on six interrelated components. These components are: disease and disorder; functions and structures; activities or limitations of activities to perform a task or action by an individual; participation or its limitations in the involvement in a life situation; environmental factors; and personal factors [12]. The purposes of ICF are very near to the purpose of this research and therefore, ICF may be suitable as a conceptual framework. A difficulty of interpreting definitions within this model may be that the ICF is generic to all health related topics and may not be sufficiently operationally defined for the use in specific working areas such as FCE. Therefore, with regards to operational definitions in FCE, widely consented definitions of experts may be very valuable because the integration of knowledge from researchers and clinicians can form a solid basis. Clear operational definitions may enhance establishment of common language and improve comparison of data. The objectives of this study were: to gain consensus in operational definitions used in FCE and to gain consensus in a conceptual framework in which FCE can be classified.

\section{Methods}

\section{Study Outline}

To reach consensus on operational definitions used in FCE and on conceptual framework, a Delphi study design was used. In total, three Delphi rounds were held. A focus group meeting was held prior to the Delphi Survey. The subsequent steps which were followed were adapted from Fowles [13] and are presented in a flow chart in Fig. 1. The first step in the process of the construction of the questionnaire was made by the authors who pre defined operational definitions that were frequently used in the international literature or operationally defined by a dictionary. Pre definitions were send to Dutch FCE experts and following to this, a Focus Group meeting was held with Dutch FCE experts in order to construct a semi structured questionnaire in which all relevant objectives were addressed. This led to the basis of the first round questionnaire. Consensus was operationally defined when $75 \%$ or higher of the participants agreed [14]. All questions on which no consensus was reached as a result of the first round, were adapted and rewritten by the authors based on recommendations of experts and were provided in the second round. An additional third round was held to address definitions in which no consensus was reached in the first two rounds. All questionnaires were sent by e-mail. Experts were given 2 weeks to fill in and return the questionnaire by e-mail or fax. E-mail reminders were sent after the first and after the second week.

\section{Participants: The Expert Panel}

The Focus Group, which was held prior to the Delphi Survey, consisted of six Dutch FCE experts and one expert of the ICF. The aim of the Focus Group was to construct a first round questionnaire and to select experts in the field of FCE. Experts were invited to represent a variety of expertise in FCE. Experts represented clinical practice, research or provider of FCE, and were working in 
Fig. 1 Flow chart of Delphi Survey

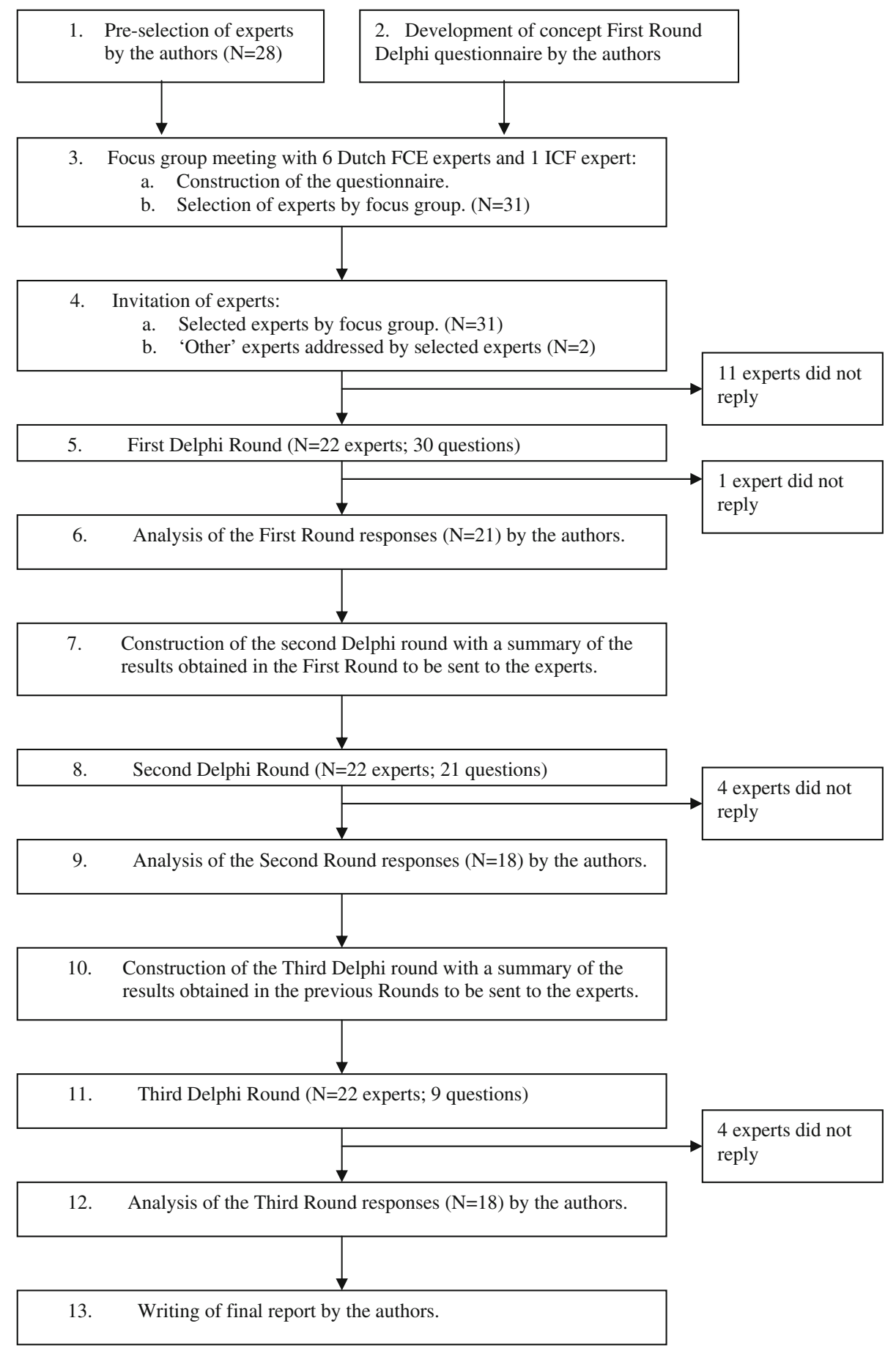

insurance, rehabilitation, occupational medicine, and education. Experts were selected if they met any of the following criteria: At least one international publication as first author and one as co-author in the field of FCE; or an individual who had developed an FCE that was subject of investigation in at least one publication in international literature. The authors consulted the Medline database to identify potential participants. Additionally, Focus Group members and invited experts were sent a list of all potentially eligible experts and they were asked whether anyone should be on the invitation list that was not invited yet but did meet the inclusion criteria. Experts who were willing to participate signed informed consent and returned this. A total of 33 potential eligible experts from North America, Australia, Asia and Europe were identified and invited to participate in this study. Anonymity of experts was guaranteed. All correspondence concerning the study was collected by the author's secretary and results were 
blinded for the authors. Data analyses were thus performed anonymously.

\section{First Round}

The first round questionnaire was semi-structured and consisted of two sections addressing 30 questions. The purpose of the first round was to explore the experts' opinions about definitions of FCE-related terms and to explore whether a conceptual framework could be used to classify terms. Additionally, experts were asked to provide additional definitions of terms besides those that were already addressed. The first section addressed the place of FCE in a conceptual framework. Section 2 of the questionnaire addressed operational definitions of FCE related terms. The content of the first round is presented in Appendix. The questionnaire took approximately $1 \mathrm{~h}$ to complete.

\section{Second Round}

Based on the results of the first round, the second round questionnaire was constructed (Appendix). This questionnaire contained 21 full structured questions. The questionnaire contained two sections. The first section addressed the place of FCE within the ICF. Experts were asked whether they did or did not concur with FCE related definitions as predefined by the ICF and whether they agreed or did not agree with certain statements used in FCE language. In section 2 of the questionnaire, experts were asked to agree or disagree with operational definitions which were used commonly in FCE. Terms indicated in Round 1 were included in the second round.

\section{Third Round}

A third round with nine questions was held to clarify different constructs in which no consensus was reached (Appendix). This questionnaire contained questions in which two definitions were proposed which were mostly supported in the second round. Additionally, this questionnaire contained questions concerning the place of FCE in ICF. Experts were given the opportunity to 'agree' or 'disagree' with a statement or to choose one definition which should be used in FCE in their opinion.

\section{Results}

A total of 33 potential eligible experts from six different countries were identified and invited to participate in this Delphi Survey. A total of 22 experts responded to this invitation and signed and returned informed consent. There were 11 non-responders (33\%). Included experts (18 researchers, 4 developers) were from Australia $(n=3)$, Europe $(n=10)$ and North America $(n=9)$.

\section{First Round}

Of all included experts, 95\% returned the questionnaire within two weeks (21 out of 22). In Tables 1 and 2, the items on which consensus was met are presented. While experts agreed upon the use of ICF as a conceptual framework, there was at this stage no consensus on how to do this. Additional definitions of terms were proposed in a high variety by the expert panel, indicating that experts are

Table 1 Items in which consensus was met concerning FCE within the framework of the ICF (agreement $\geq 75 \%$ )

\begin{tabular}{|c|c|c|}
\hline $\begin{array}{l}\text { Delphi round; } \\
\text { Item number }\end{array}$ & Statement & Agreement $(\%)$ \\
\hline $1 ; 3$ & ICF may offer a suitable classification to operationally define terms used in FCE & 77 \\
\hline $1 ; 2$ & FCE should not focus on disability solely & 100 \\
\hline $1 ; 1$ & FCE should at least measure activities at the level of the whole person & 95 \\
\hline $2 ; 3$ & $\begin{array}{l}\text { FCE is performance based measurement to determine what the person can do safely, not } \\
\text { what he/she can't do }\end{array}$ & 89 \\
\hline $2 ; 1$ & $\begin{array}{l}\text { FCE primarily focuses on activity level which include complex interactions between } \\
\text { the domains body functions/structures and activities and participation }\end{array}$ & 83 \\
\hline $2 ; 2$ & $\begin{array}{l}\text { The purpose of FCE is to make decisions on the level of functioning (acting in his/her } \\
\text { environment) }\end{array}$ & 78 \\
\hline $2 ; 8$ & $\begin{array}{l}\text { The capacity qualifier according to the ICF represents more than 'the maximum anatomical } \\
\text { limits of a person', because mental functions and personal factors are also of influence } \\
\text { in the capacity qualifier }\end{array}$ & 83 \\
\hline $2 ; 9$ & Anatomical limits are a part of the capacity qualifier & 100 \\
\hline $3 ; 8$ & $\begin{array}{l}\text { The difference between a Work Capacity Evaluation and a Functional Capacity Evaluation } \\
\text { is not the setting but the content of the tasks. We can describe these differences in work } \\
\text { related tasks and in functional tasks }\end{array}$ & 77 \\
\hline
\end{tabular}

FCE functional capacity evaluation, ICF international classification of functioning disability and health 
Table 2 Items in which consensus was met concerning operational definitions of FCE related terms (agreement $\geq 75 \%$ )

\begin{tabular}{|c|c|c|}
\hline $\begin{array}{l}\text { Round; Item } \\
\text { number }\end{array}$ & Definition & $\begin{array}{l}\text { Agreement } \\
(\%)\end{array}$ \\
\hline $1 ; 10$ & $\begin{array}{l}\text { Evaluation is 'a systematic approach including observation, reasoning and conclusion. Going beyond } \\
\text { monitoring and recording, the evaluation process implies an outcome statement that is } \\
\text { explanatory, as well as an objective measurement' }\end{array}$ & 82 \\
\hline $1 ; 13$ & Test is 'a standardized procedure of measurement' & 86 \\
\hline $1 ; 23$ & $\begin{array}{l}\text { Inconsistency is 'lacking agreement, as one thing with another or two or more things in relation to } \\
\text { each other' }\end{array}$ & 95 \\
\hline $2 ; 4$ & Performance is 'what a person does in the current environment' ${ }^{\mathrm{a}}$ & 83 \\
\hline $2 ; 7$ & $\begin{array}{l}\text { Capacity is 'the highest probable level of functioning that a person may reach in a domain at a } \\
\text { given moment in a standardized environment'a }\end{array}$ & 78 \\
\hline $2 ; 21$ & Injury is 'damage or harm done to or suffered by a person or thing'b & 89 \\
\hline $3 ; 3$ & Screening is 'a review to see if further evaluation is needed. It is not a full evaluation itself' & 88 \\
\hline $3 ; 4$ & $\begin{array}{l}\text { Evaluation and assessment are the preferred terms to be used in FCE. One can use either one of } \\
\text { them, depending on the purpose }\end{array}$ & 83 \\
\hline $3 ; 5$ & Assessment is 'a systematic approach including observation, reasoning and conclusion' & 87 \\
\hline $3 ; 9$ & $\begin{array}{l}\text { Safety is 'a situation that, given the known characteristics of the person, the procedure should not } \\
\text { be expected to lead to injury' }\end{array}$ & 78 \\
\hline
\end{tabular}

FCE functional capacity evaluation; ICD international classification of diseases

${ }^{\mathrm{a}}$ ICF definition; ${ }^{\mathrm{b}}$ ICD-10 definition

using different terms and definitions of terms. Three items were accepted concerning conceptual framework (Table 1) and three definitions were accepted (Table 2). As a result of the first round, the authors chose to exclude further questions concerning work performance, work ability, work tolerance, malingering and aggravation. Authors did this because the experts agreed with the complexity and extensiveness of these terms and should be researched separately from this study (see Table 3 ).

\section{Second Round}

The response rate after the second round was $82 \%$ (18 out of 22) in this round. Experts reached consensus on the definitions as they were predefined within the ICF. Five items were accepted concerning conceptual framework (Table 1) and three definitions were accepted (Table 2).

\section{Third Round}

The response rate of the third round was $82 \%$. Consensus was being reached in five out of nine questions. Results of the third round are presented in Tables 1 and 2. After the third round, consensus was reached on 19 items. Nine items represented operational definitions and ten items concerned the place of ICF in a conceptual framework.

No consensus was reached on nine definitions, on which five were excluded as a result of the first round. Definitions

Table 3 FCE related items in which no consensus was met

\begin{tabular}{ll}
\hline Item & Reason \\
\hline Work performance & Excluded after round 1. Term 'work' is too extensive; scope is beyond \\
FCE and should be studied in a separate study \\
Work ability & FCE and should be studied in a separate study \\
& Excluded after round 1. Term 'work' is too extensive; scope is beyond \\
Work tolerance & FCE and should be studied in a separate study \\
Malingering & Excluded after round 1. No consensus reached; no alternative definitions proposed \\
Recovery & Excluded after round 1. Relation of item deemed to far from FCE \\
Aggravation & Excluded after round 1. No consensus reached; no alternative definitions proposed \\
Functional capacity evaluation & No consensus reached \\
Physical capacity evaluation & No consensus reached \\
Work capacity evaluation & No consensus reached \\
Ability & No consensus reached
\end{tabular}

FCE functional capacity evaluation 
for which no consensus was reached after the third round were: FCE; Physical Capacity Evaluation; recovery and ability. All excluded definitions are presented in Table 3.

\section{Discussion}

One of the main results of this study was that experts agreed on using the ICF as a conceptual framework for FCE and that experts consented with definitions of terms as defined in the ICF. The study results gain more insight in the definitions which are used frequently in FCE and contribute therefore to psychometric characteristics of FCEs. Interestingly, no consensus was reached on the term FCE itself. Even though consensus was reached on the different terms that comprise FCE, no consensus was reached for one single definition of FCE. It appears that this combination of terms seem to be interpreted differently than the items solely. After elimination of optional definitions during three rounds, two definitions remained. The two definitions with the highest points scored were:

1. A FCE is an evaluation designed to document and to describe a person's current safe work ability from a physical ability and motivational perspective with consideration given to any existing medical, impairment and/or pain syndromes. (38\% agreement)

2. A FCE is an evaluation of capacity of activities that is used to make recommendations for participation in work while considering the person's body functions and structures, environmental factors, personal factors and health status. (63\% agreement)

In both definitions, multiple biopsychosocial factors such as personal and contextual factors are taken into consideration. Moreover, both definitions consider similarity of FCE purpose, namely to evaluate ability or participation for work. It remains unclear whether both definitions can be compared to each other on outcome because no consensus was reached on the term ability. Both definitions may be not mutually exclusive and some experts stated that they may be even complementary to each other. However, if ICF were to be used as a framework for FCE, the second definition seems preferable because all terms are defined within the ICF. However, as authors of this study, we have excluded ourselves as participants in this study. Therefore, based on the predefined methodology of this study, we cannot recommend one definition over the other. Thus, it is recommended that in future studies researchers provide the definition of FCE they used.

Former research to psychometric properties of functional tests had recommended that all test selection should be done based to psychometric properties of safety, reliability, validity, practicality, and utility [15]. Safety, for example, has previously been object of discussion, merely, because of the lack of a consented operational definition for safety and for injury [16-18]. Therefore, above all, previously mentioned properties can only be applied when measurement instruments are placed and described in the context in which they are intended to and if operational definitions are clear. This is a crucial point in many different health sectors in which researchers from different areas conflict with each other because of a lack of consensus in terminology. This, in turn, makes it impossible to compare or interpreted data correctly and seriously hinders progression in this field. ICF may in this case offer a framework in which multiple work fields may classify definitions [12]. ICF, however, is universal to all health related sectors and should in most cases be further operationally defined to be of use in other specific sectors.

A difficulty in this study was, as mentioned above, that ICF is generic to all health related sectors and not specific to any work field in particular. FCE development evolved in the 1980s, 20 years before the introduction of the ICF in 2001 [12]. This made it difficult to post hoc classify terminology in a framework of a date beyond introduction of FCE. Another difficulty in reaching consensus was the differences of work disciplines and health disciplines involved in FCE. Therefore, an expert group was selected which consisted of persons working in insurance medicine, rehabilitation medicine, occupational medicine, and education. Because FCE is used by different disciplines, terminology had evolved in the past decades to a jumble of terms in which different health care providers used different terms. In the 1980s, FCE developers and researchers were strongly influenced by the biomedical model. The term capacity, for example, was first defined as 'physical abilities maximums' [19]. This dualistic approach formed a basis of categorization of physical and psychosocial factors influencing the individual based on body functions and structures. This approach excludes contextual or personal factors by stating that functioning is no more than the sum of different body functions or structures. As a result of the first round, experts agreed on using the ICF as a potential useful classification system for FCE and related terminology. The experts disagreed, however, on how to classify the terms of 'capacity' and 'performance'. There appeared to be a rather strict separation between biomedical oriented and bio psychosocial oriented experts. Where the biomedical oriented defined capacity as "the maximal limits of the anatomical system", the bio psychosocial oriented [20] disagreed because we cannot measure the maximal limits of the anatomical system and capacity is about functioning and not about body functions/structures. The latter agree with a definition such as 'the highest probable level of functioning'. The main result of the first round was 
therefore: experts do agree that the ICF provides a useful framework but do not agree on how to classify definitions within the ICF.

One objective of the second round was to confront experts with this contrast. Authors constructed a questionnaire to address these issues. All experts were asked whether they could or could not concur with the definitions of capacity and performance as they were predefined within the ICF. At least $79 \%$ of all experts concurred with these questions. Some experts who did not concur with these definitions did this because "FCE terminology had already been developed in the biomedical context and was not incorporated in the ICF model."

A general weakness of this study may be selection bias of included experts. Some experts may have dropped out or resign to participate because of negative feelings they have about the study. The response rate of all experts who agreed to participate, however, was above $80 \%$ in all three rounds. Delphi studies, however, have been found an effective way to gain and measure group consensus in healthcare [21]. To reduce the risk of excluding experts who should have been included in this study, the focus group, which was held prior to the Delphi Survey, was asked whether any experts should be invited who was not pre-screened from the Medline database by the authors. This resulted in three additional experts. This question was again asked to all experts when sending the first invitation. Again, two additional experts were included. Nevertheless, experts could have been missed which may have led to a selection bias. Another point of selection bias was present because two of the authors of this study (RS; MR) met the inclusion criteria for experts but were not included in the expert panel. Strength of this study was that experts did not directly interact with each other, which prevented social processes or contaminations that can happen in group processes. Where single experts may suffer biases and group meetings suffer from 'follow the leader' tendencies, a Delphi method was assumed to be the most appropriate technique for this consensus study [22].

In conclusion, the results of this study show that consensus was reached in a large part of operational definitions in FCE. This may enable researchers as well as clinicians to improve communication and to better interpret data and patient outcome. In this study, consensus was met on using the ICF as a conceptual framework in order to classify terminology of FCE. Experts met consensus to use predefined terms of the ICF. Consensus was met in 19 statements and definitions in total. No consensus was met about a definition of FCE for which two potential eligible definitions remained. It was recommended that authors define definitions they use in future research in order to permit comparison of data and to serve as the use of a common language.
Acknowledgments The authors are grateful to all who contributed to this study. Our thanks go to the study secretary, Mrs. Marleen Speller; Participants of the focus group: Sandra Brouwer, Vincent Gouttebarge, Paul Kuijer, Haje Wind, and Harriet Wittink; ICF experts: Yvonne Heerkens and Gonda Stallinga; The FCE expert panel: Lisa Blankenship (representing Blankenship FCE), Sandra Brouwer, Monique Frings-Dresen, Michael Geisser, Libby Gibson (Both researcher and representing GAPP FCE), Vincent Gouttebarge, Douglas Gross, Gary Harbin, Dennis Hart, Ev Innes, Susan Isernhagen, Jan Kool, Wietske Kuijer, Len Matheson, Margot Miller (representing WorkWell FCE), Vert Mooney, Peter Oesch, Jan Plat, (representing Ergokit FCE), Glen Pransky, Maureen Simmonds, Haije Wind, and Harriet Wittink.

Open Access This article is distributed under the terms of the Creative Commons Attribution Noncommercial License which permits any noncommercial use, distribution, and reproduction in any medium, provided the original author(s) and source are credited.

\section{Appendix}

Questionnaires Used in Delphi Study

\section{Round 1}

1. In your opinion; which level of ICF should FCE address? (Multiple answers possible)

(1) Body function and body structure level,

(2) Activities at the level of the whole person,

(3) Participation at the level of the whole person in a social context

(4) No opinion

2. In your opinion; should FCE focus on functioning, disability or both?

3. In your opinion; The ICF may offer a suitable classification to operationally define terms used in FCE:

4. Please indicate whether you agree with the following definitions of Capacity for FCE

- An individual's ability to execute a task or an action. This construct identifies the highest probable level of functioning of a person in a standardized or ideal environment at a given moment.

- The maximal limits of an individual in terms of anatomical, physiological and psychological systems.

5. Ability is a not defined term in the ICF but is frequently used as the opposite of disability.

In your opinion: ability should be operationally defined as being equal to functioning (as defined in the ICF). 
6. If you (strongly) disagree with question 5; please indicate whether you agree with the following definitions of Ability for FCE:

- The quality of being able to perform tasks, especially the physical, mental, financial, or legal power to accomplish these tasks.

- Human capacities which are modified by an individual's attitudes, injury and pain, as well as by environmental factors, such as physical and social stressors.

7. Please indicate whether you agree with the following definitions of Performance:

- What an individual does in his or her current environment. Because the current environment brings in a societal context, performance as recorded by this qualifier can also be understood as "involvement in a life situation". The current environment will be understood to include assistive devices or personal assistance.

- The act or process of functioning in any environment.

8. ICF makes no difference between "that what a person does in the FCE setting", and "that what a person could do in the FCE setting." To enable us to make this difference and to appreciate the specific environmental context in which an FCE takes place (lab situation), other definitions of capacity and performance may be better.

- Capacity in the FCE context should be defined as "the maximal limits of an individual"

- Performance in the FCE context should be defined as "that what a person does in the current environment (including the FCE setting)?"

9. In your opinion, is performance or capacity measured with an FCE?

(1) Performance following the definition of 7A

(2) Performance following the definition of $7 \mathrm{~B}$

(3) Performance following the definition of $8 \mathrm{~B}$

(4) Capacity following the definition of $4 \mathrm{~A}$

(5) Capacity following the definition of $4 \mathrm{~B}$

(6) Capacity following the definition of $8 \mathrm{~A}$

(7) No opinion

10. Please indicate whether you agree with the following definitions of Evaluation:

- A diagnosis or diagnostic study of a physical or mental condition.
- The process of obtaining and interpreting data necessary for understanding the individual, system or situation.

- A 'systematic approach including observation, reasoning and conclusion.' Going beyond monitoring and recording, the evaluation process implies an outcome statement that is explanatory, as well as an objective measurement.

- Proposed alternative definition of Evaluation: No/ Yes. If yes, please define: ...

11. Please indicate whether you agree with the following definitions of Assessment:

- Specific tools, instruments, or interactions used during the evaluation process with comparison of the affected body part to the norm (a component of the evaluation).

- The process of investigating an individual's ability and disability with respect to expected levels of performance.

- Proposed alternative definition of Assessment: No/Yes. If yes, please define: ...

12. Please indicate whether you agree with the following definitions of Screening:

- Obtaining and reviewing data relevant to a potential patient to determine the need for further evaluation and intervention.

- The presumptive identification of unrecognized disease or defect by the application of tests, examinations or other procedures which can be applied rapidly.

- Proposed alternative definition of Screening: No/ Yes. If yes, please define: ...

13. Please indicate whether you agree with the following definition of Test:

- Standardized procedure of measurement.

- Proposed alternative definition of Test: No/Yes. If yes, please define: ...

14. Scientific literature uses different terms to define the action taken in FCE such as screening, assessment, analyses, and evaluation.

- In your opinion, which one should preferably be used in FCE?

(1) Screening

(2) Assessment

(3) Test

(4) Evaluation

(5) No opinion 
15. Please indicate whether you agree with the following definitions of FCE:

- Performance based evaluation about an individuals' capacity for work, thereby enabling appropriate decision-making with respect to future management of the injured worker.

- Batteries of tests to measure the ability to perform work-related activities.

- Functional task to determine a worker's sincerity of effort.

- FCE are supposed to define an individual's functional abilities or limitations in the context of safe productive work tasks.

- What a person can and cannot do.

- Proposed alternative definition of FCE: No/Yes. If yes, please define: ...

16. Please indicate whether you agree with the following definition of Physical Capacity Evaluation:

- A one time evaluation, using measures to determine the maximal performance of isolated physical attributes.

- Proposed alternative definition of Physical Capacity Evaluation: No/Yes. If yes, please define: ...

17. Please indicate whether you agree with the following definition of Work performance:

- The process of functioning over a period of time in the presence of various environmental factors and stressors (e.g., heat, humidity, time pressure), and individual characteristics.

- Proposed alternative definition of Work performance: No/Yes. If yes, please define: ...

18. Please indicate whether you agree with the following definition of Work ability:

- A match of functioning and required environmental demands in the work situation.

- Proposed alternative definition of Work ability: No/Yes. If yes, please define: ...

19. Please indicate whether you agree with the following definition of Work tolerance:

- The observed and measured physical competencies to perform the physical demands of work tasks. Measured as the ability to sustain a given work effort at a prescribed frequency over a given period of time.

- Proposed alternative definition of Work tolerance: No/Yes. If yes, please define: ...

20. Please indicate whether you agree with the following definitions of Safety:
- A situation that, given the known characteristics of the person, the procedure should not be expected to lead to injury.

- Proposed alternative definition of Safety: No/Yes. If yes, please define: ...

21. Please indicate whether you agree with the following definitions of Injury:

- Damage or harm done to or suffered by a person. (Harm: physical injury or mental damage.)

- A particular form of hurt, damage, or loss.

- Proposed alternative definition of Injury: No/Yes. If yes, please define: ...

22. Please indicate whether you agree with the following definitions of Malingering:

- Pretending illness when the individual has a clear motive-usually to benefit economically or to avoid legal trouble.

- Intentional production of false or grossly exaggerated physical or psychological symptoms, motivated by external incentives such as avoiding military duty, avoiding work, obtaining financial compensation, evading criminal prosecution, or obtaining drugs.

- Proposed alternative definition of Malingering: No/Yes. If yes, please define: ...

23. Please indicate whether you agree with the following definition of Inconsistency:

- Lacking agreement, as one thing with another or two or more things in relation to each other.

- Proposed alternative definition of Inconsistency: No/Yes. If yes, please define: ...

24. Please indicate whether you agree with the following definition of Aggravation:

- Action that makes a problem, a disease, (or its symptoms) worse.

- Proposed alternative definition of Aggravation: $\mathrm{No} / Y e s$. If yes, please define: ...

25. Please indicate whether you agree with the following definition of Recovery:

- Restoration or return to health from sickness.

- Proposed alternative definition of Recovery: No/ Yes. If yes, please define: ...

26. Physical Capacity Evaluations measure isolated physical attributes in the domain of body functions and structures as classified in the ICF.

27. If FCEs measure work-related activities, do you agree with the following statement? 
- FCEs measure work related activities in the domain of activities as classified in the ICF.

28. Work Performance Evaluations measure work related performance over a time in the presence of various external factors in the domain of participation as classified in the ICF.

29. FCEs are administered in the context of rehabilitation medicine, occupational medicine, vocational medicine, insurance and medico legal matters.

- In your opinion, should the terms used in FCE be defined independently of the context in which the FCE is administered?

30. Are there any questions or definitions in this questionnaire which have not been addressed and should, in your opinion, be added to the questionnaire for the second round?

\section{Round 2}

1. FCE primarily focuses on activity level which include complex interactions between the domains body functions/structures, activities and participation. (If needed see Appendix).

2. The purpose of FCE is to make decisions on the level of functioning (acting in his/her environment).

3. FCE is performance based measurement to determine what the person can do safely, not what he/she cannot do.

4. We have not reached consensus on a definition of Performance. Can you concur with the definition of Performance as given in the ICF?

ICF definition of Performance: what a person does in the current environment. The current environment can be understood as 'involvement in a life situation'. (If needed: see Appendix).

5. If you concur with the ICF definition of Performance, than the performance qualifier can only be used when measures take place in the environmental (e.g., work) context.

6. FCE is designed to measure in a standardized or uniform environment and therefore the performance qualifier is of no use in FCE.

7. We have not reached consensus on a definition of Capacity. Can you concur with the definition of Capacity as given in the ICF?

ICF definition of Capacity: the highest probable level of functioning that a person may reach in a domain at a given moment in a standardized environment. (if needed: see Appendix).
8. Do you agree with this statement?

The capacity qualifier according to the ICF represents more than the maximum anatomical limits of a person', because mental functions and personal factors are also of influence in the capacity qualifier.

9. Anatomical limits of a person are a part of the capacity qualifier.

10. When capacity is evaluated in the FCE, one can compare this result with the functional demands of the job (environmental factors) and determine the level of performance.

11. Ability is:

- The potential to function and modified by the personal factors as well as by environmental factors.

- potential to perform activities.

- Human capacities (which are modified by personal and environmental factors).

- An umbrella term for body functions, structures, activities and participation.

- The absenteeism of problems of the person which are caused by disease, trauma or other health condition.

- Full integration of individuals into society.

12. Evaluation: the name used depends on the purpose of the FCE. All can be used, depending on the purpose

13. Assessment is:

- The process of investigating an individual's ability and disability with respect to expected levels of performance.

- A systematic approach including observation, reasoning and conclusion.

- Procedure to assemble the information measuring to answer the research question.

- A data-gathering strategy, analyses and reporting process that provide information that can be used to determine whether or not intended outcomes are being achieved.

14. Evaluation uses assessment information to support decisions on maintaining, changing, or discarding instructional or programmatic practices

15. Assessment and evaluation are often used interchangeable; evaluation has tended to come from North America and Assessment is used in Australia. The difference therefore is of geographic nature

16. Screening is:

- A review to see if further evaluation is needed. It is not a full evaluation itself. 
- Procedure to rapidly search large groups on the presence or absence of pre-defined characteristics.

17. FCE is:

- An evaluation designed to document and to describe a person's current safe work ability from a physical ability and motivational perspective with consideration given to any existing medical, impairment and/or pain syndromes.

- An evaluation of capacity of activities that is used to make recommendations for participation in work while considering the person's body functions and structures, environmental, personal factors and health status.

- A detailed evaluation that objectively measures a person's current level of functioning in terms of the demands of competitive employment.

- An evaluation to determine the level of functioning of a client.

18. Physical capacity evaluation: to operationally define body functions and structures the qualifier capacity cannot be used and therefore, the term Physical Capacity Evaluation is a misnomer.

19. Work Capacity Evaluation: the difference between a work capacity evaluation and FCE is not the setting but the content of the tasks. We can describe these in differences in work related tasks and in functional tasks (which are work related and not work related).

20. Safety:

- The condition of being protected against injury

- A situation that, given the known characteristics of the person, the procedure should not be expected to lead to symptoms or injury

- A situation that no impairment will occur

- A situation that, given the known characteristics of the person, the procedure should not be expected to lead to injury

21. Injury: damage or harm done to or suffered by a person or thing.

\section{Round 3}

1. The purpose of FCE is to determine the level of performance by comparing functional demands of the job with evaluated capacity of a person.

2. Ability:

The two definitions of ability with the highest points from the second round are presented below. Please choose one definition that you think should be used for FCE.
- Ability is the potential to function and modified by the personal factors as well as by environmental factors.

- Ability is the potential to perform activities.

3. Screening:

The two definitions of screening with the highest points as a result from the second round are presented below.

- Screening is a review to see if further evaluation is needed. It is not a full evaluation itself.

- Screening is a procedure to rapidly search large groups on the presence or absence of predefined characteristics.

The experts were not exclusive about one definition and many divided their 10 points to both definitions. Perhaps both definitions may apply to different types of screening and are therefore both correct. Definition $a$, however, may be suitable for FCE because definition $b$, refers explicitly to screening of large groups.

Do you agree with the authors that definition $a$. is a suitable definition for the use in FCE?

4. Scientific literature uses different terms to define the action taken in FCE such as screening, assessment, analyses and evaluation. Do you agree with the following statement?

Evaluation and assessment are the preferred terms to be used in FCE. One can use either one of them, depending on the purpose.

5. Assessment:

The two definitions of assessment with the highest points from the second round are presented below. Please choose one definition that you think should be used for FCE.

- Assessment is the process of investigating an individual's ability and disability with respect to expected levels of performance.

- Assessment is a systematic approach including observation, reasoning and conclusion.

6. FCE:

The two definitions of FCE with the highest points from the second round are presented below. Please choose one definition that you think should be used for FCE.

- A FCE is an evaluation designed to document and to describe a person's current safe work ability from a physical ability and motivational 
perspective with consideration given to any existing medical, impairment and/or pain syndromes.

- A FCE is an evaluation of capacity of activities that is used to make recommendations for participation in work while considering the person's body functions and structures, environmental factors, personal factors and health status.

\section{Physical Capacity Evaluation:}

You have not reached consensus on a definition of Physical Capacity Evaluation. Do you agree with this statement?

Physical Capacity Evaluation is misplaced because the term physical refers to body functions and structures.

\section{Work Capacity Evaluation:}

The difference between a Work Capacity Evaluation and FCE is not the setting but the content of the tasks. We can describe these in differences in work related tasks and in functional tasks.

\section{Safety:}

The two definitions of Safety with the highest points from the second round are presented below. Please choose one definition that you think should be used for FCE.

- A situation that, given the known characteristics of the person, the procedure should not be expected to lead to symptoms or injury.

- A situation that, given the known characteristics of the person, the procedure should not be expected to lead to injury.

\section{References}

1. Gouttebarge V, Wind H, Kuijer PP, Frings-Dresen MH. Reliability and validity of functional capacity evaluation methods: a systematic review with reference to Blankenship system, ERGOS work simulator, Ergo-Kit and Isernhagen work system. Int Arch Occup Environ Health. 2004;77(8):527-37. doi:10.1007/s00420004-0549-7.

2. Rustenburg G, Kuijer PP, Frings-Dresen MH. The concurrent validity of the ERGOS work simulator and the Ergo-Kit with respect to maximum lifting capacity. J Occup Rehabil. 2004; 14(2):107-18. doi:10.1023/B:JOOR.0000018327.27554.58.

3. Soer R, Poels BJ, Geertzen JH, Reneman MF. A comparison of two lifting assessment approaches in patients with chronic low back pain. J Occup Rehabil. 2006;16(4):639-46. doi:10.1007/ s10926-006-9055-y.
4. IJmker S, Gerrits EH, Reneman MF. Upper lifting performance of healthy young adults in functional capacity evaluations: a comparison of two protocols. J Occup Rehabil. 2003;13(4):297305. doi:10.1023/A:1026229006792.

5. Reneman MF, Kool J, Oesch P, Geertzen JH, Battie MC, Gross DP. Material handling performance of patients with chronic low back pain during functional capacity evaluation: a comparison between three countries. Disabil Rehabil. 2006;28(18):1143-49. doi:10.1080/09638280600551427.

6. King PM, Tuckwell N, Barrett TE. A critical review of functional capacity evaluations. Phys Ther. 1998;78(8):852-66.

7. Innes E, Straker L. A clinician's guide to work-related assessments: 2-design problems. Work (Reading, Mass.). 1998;11(2): 191-206. doi:10.1016/S1051-9815(98)00037-0.

8. Wittink H. Functional capacity testing in patients with chronic pain. Clin J Pain. 2005;21(3):197-9. doi:10.1097/00002508-200 505000-00001.

9. Gibson L, Strong J. A conceptual framework of functional capacity evaluation for occupational therapy in work rehabilitation. Aust Occup Ther J. 2003;50(2):64-71. doi:10.1046/j.14401630.2003.00323.x.

10. Gross DP. Measurement properties of performance-based assessment of functional capacity. J Occup Rehabil. 2004;14(3): 165-74. doi:10.1023/B:JOOR.0000022759.30446.4f.

11. Stamm TA, Cieza A, Machold KP, Smolen JS, Stucki G. Content comparison of occupation-based instruments in adult rheumatology and musculoskeletal rehabilitation based on the international classification of functioning, disability and health. Arthritis Rheum. 2004;51(6):917-24. doi:10.1002/art.20842.

12. WHO.ICF. International classification of functioning, disability and health. Geneva: World Health Organization; 2001.

13. Fowles J. Handbook of futures research. Westport: Greenwood; 1978.

14. Miro J, Nieto R, Huguet A. Predictive factors of chronic pain and disability in whiplash: a Delphi poll. Eur J Pain (London, England). 2008;12(1):30-47. doi:10.1016/j.ejpain.2007.02.004.

15. Hart DL, Isernhagen SJ, Matheson LN. Guidelines for functional capacity evaluation of people with medical conditions. J Orthop Sports Phys Ther. 1993;18(6):682-6.

16. Gibson L, Strong J. Safety issues in functional capacity evaluation: findings from a trial of a new approach for evaluating clients with chronic back pain. J Occup Rehabil. 2005;15(2):237-51. doi:10.1007/s10926-005-1222-z.

17. Reneman MF, Kuijer W, Brouwer S. Item validity of the physical demands from the dictionary of occupational titles for FCE of clients with chronic back pain. Work (Reading). 2006;26(2):229.

18. Reneman MF, Kuijer W, Brouwer S, Preuper HR, Groothoff JW, Geertzen JH, et al. Symptom increase following a functional capacity evaluation in patients with chronic low back pain: an explorative study of safety. J Occup Rehabil. 2006;16(2):196205. doi:10.1007/s10926-006-9023-6.

19. Isernhagen SJ. Functional capacity evaluation: rationale, procedure, utility of the kinesiophysical approach. J Occup Rehabil. 1992;2(3):157-68. doi:10.1007/BF01077187.

20. Engel GL. The need for a new medical model: a challenge for biomedicine. Science. 1977;196(4286):129-36. doi:10.1126/ science. 847460 .

21. Murphy MK, Black NA, Lamping DL, McKee CM, Sanderson $\mathrm{CF}$, Askham J, et al. Consensus development methods, and their use in clinical guideline development. Health Technol Assess. 1998;2(3):1-88.

22. Gatewood RD, Gatewood EJ. The use of expert data in human resource planning: guidelines from strategic forecasting. Hum Resour Plan. 1983;5(1):83-94. 